\title{
Estudio observacional sobre presencia y caracterización de hematomas en carcasas vacunas de Uruguay
}

\author{
Gonzalo Crosi ${ }^{1 *}$; Martín Prado ${ }^{1}$; Stella Huertas ${ }^{2}$; Juan Imelio ${ }^{2}$; José Piaggio ${ }^{1}$; Andres Gil ${ }^{2}$ \\ ${ }^{1}$ Ministerio de Ganadería Agricultura y Pesca, Uruguay \\ ${ }^{2}$ Facultad de Veterinaria-Universidad de la República, Uruguay \\ *gonzalocrosim@gmail.com \\ Aceptado para publicación: 01 de Octubre de 2015
}

\begin{abstract}
RESUMEN
Objetivo: Evaluar y caracterizar los hematomas presentes en vacunos faenados en las plantas frigoríficas de Uruguay, habilitadas para exportación. Comparar datos obtenidos con investigaciones anteriores a nivel nacional. Metodología: Se realizó un estudio observacional de las carcasas bovinas luego de la faena, con el fin de evaluar la presencia de hematomas, teniendo en cuenta su localización, área, forma y profundidad. A su vez, se recabó información sobre el sexo y categoría de las tropas evaluadas, de manera de correlacionar estos factores con la aparición de las lesiones. Resultados y conclusiones: De un total de 1.030 carcasas observadas, $44.4 \%$ presentaron al menos un hematoma, con un promedio de 0.89 hematomas por carcasa. Mayormente se localizaron en la región del muslo, costillar y flanco, con $31 \%$, $19.7 \%$ y $19.6 \%$ respectivamente. En cuanto a la profundidad, $83.9 \%$ de las carcasas lesionadas presentaron contusiones grado 1 o superficial y $16.1 \%$ grado 2, destacando la ausencia de hematomas grado 3. Los hematomas "circulares o irregulares" fueron los más frecuentemente registrados, detectando también formas "lineales", en "tranvía" y en forma de "coma". Por otra parte, se relacionaron las lesiones con factores del propio animal, encontrando diferencias significativas $(p<0.01$ ) según su edad y sexo. Las hembras presentaron más hematomas (54\%) que los machos (37\%), mientras que fue mayor la cantidad de lesiones halladas en los animales adultos $(55.9 \%)$ a lo encontrado en jóvenes (37.4\%). La presencia de lesiones, si bien ha disminuido en el correr de los años, implica que aún existen factores durante la vida del animal que influyen negativamente en su nivel de bienestar. Son necesarias nuevas estrategias o perfección de las ya utilizadas, con el objeto de lograr un desarrollo continuo y obtener así un producto de buena calidad desde el punto de vista del bienestar animal. Palabras clave: Bienestar animal, bovino, carcasa, hematoma, calidad de carne.
\end{abstract}

\begin{abstract}
Aim: To assess and characterize bruises present in cattle slaughtered in Uruguayan plants, authorized to export. Compare the results with previous national studies. Methods: An observational study of bovine carcasses after slaughter was performed, in order to assess the presence of bruises, considering its location, area, shape and deepness. Furthermore, information about sex and age of animals was collected, to correlate these factors with the traumatic injuries. Results and conclusions: 1.030 carcasses were observed, $44.4 \%$ had at least one lesion, with an average of 0.89 bruise per carcass. Most of the bruises were located in the region of the buttock, rib and hip, with $31 \%, 19.7 \%$ y $19.6 \%$ respectively. As to the deepness, $83.9 \%$ of the injured carcasses had bruises grade 1 or superficial, meanwhile, $16.1 \%$ had deeper bruises grade 2 , with an absence of carcasses showing severe bruises, classified as grade 3. The "circular or irregular" bruises were the most frequently recorded, noting also lineal bruises, those like a tram-line and comma-shaped bruises. Moreover, relating the animal factors with the bruises, it was found that exist significant differences $(\mathrm{p}<0.01)$ according to their age and sex. Females showed more bruises $(54 \%)$ than males $(37 \%)$, while it was higher the number of lesions in adults $(55.9 \%)$, compared with young animals $(37.4$ $\%$ ). The presence of bruises, even though it is lower, there are still factors in the life of the animals that affects negatively their welfare. It is necessary to look for new strategies in order to achieve a constant development, and so be able to obtain a quality product from the animal welfare point of view.
\end{abstract}

Key words: Animal welfare, bovine, carcass, bruise, meat quality. 


\section{INTRODUCCIÓN}

Hematomas como indicadores de bienestar animal y calidad de la carne

La calidad de la carne es influenciada por factores tanto endógenos como ambientales, como son las condiciones ante-mortem y post-mortem. Los manejos que se realizan en el ganado destinado a producir carne en las horas previas a su sacrificio son de los más estresantes en su vida (Castro \& Robaina, 2003; Huertas, 2004; Gallo, 2004). Esa sucesión de eventos donde los animales son constantemente movilizados, mezclados con otros animales desconocidos para ser enviados a las plantas de faena, redunda en pérdidas de peso, alteraciones en la calidad de la carne (Huertas, 2004), y en el peor de los casos, hasta la muerte de los animales por el transporte inadecuado o con ayuno prolongado. Por lo tanto, los manejos inadecuados durante el período previo a la faena pueden tener consecuencias de importancia económica, ya que la calidad de un producto y la cantidad, están directamente vinculadas con su valor económico. (INAC, 2003).

Un factor que influye en gran medida sobre las pérdidas económicas que ocurren en la industria cárnica por decomisos (eliminación de zonas de carne dañadas), es la aparición de hematomas en las carcasas. Estos, son lesiones causadas por un daño mecánico de los tejidos, a causa de un golpe que provoca la ruptura de los vasos sanguíneos, con salida de sangre y suero hacia los tejidos circundantes provocando dolor e hinchazón (Hoffman et al., 1998, Gracey, et al., 1999).

Los hematomas en el bovino son detectados postmortem en las carcasas, luego de la faena del animal. Sin embargo, tienen su génesis ante-mortem, pueden ocurrir en cualquier etapa de la cadena y el factor humano es de los más contribuyentes a su aparición. El uso y abuso de herramientas para obligar a avanzar al ganado, así como el diseño incorrecto y la falta de mantenimiento de las instalaciones, tanto en los establecimientos ganaderos, vehículos de transporte y plantas de faena, son ejemplos de sus eventos causales.

La generación de estas contusiones tiene un impacto negativo en el nivel de bienestar animal, debido a que implican dolor y sufrimiento. Por lo tanto, su hallazgo post-mortem reflejan las pobres condiciones a las que fueron sometidos los animales en vida (Strappini et al., 2009). Por otra parte, constituyen un riesgo para la salud pública debido a su capacidad de actuar como medio propicio para la proliferación y multiplicación de potenciales patógenos. No pueden ser utilizadas en la preparación de carnes procesadas, y en cuanto a su aspecto visual, son inaceptables para el consumidor. Por estas razones, constituyen un material de decomiso, todo el tejido dañado y gran parte del tejido circundante debe ser removido de la carcasa antes de ser considerada como alimento para consumo humano (Marshall, 1977; FAO, 2001). Su significado económico se ve reflejado en las pérdidas cuantitativas por la remoción de la carne dañada y por la reducción del valor comercial que tendrá esa canal (Cervieri et al., 2010; Strappini et al., 2011).

El estudio de las características (localización anatómica, severidad y apariencia) de los hematomas varía extensamente y pueden brindar información de alto valor para la determinación de la causa de la lesión (Hoffman \& Lühl, 2012).

En Uruguay, la presencia de contusiones en las carcasas es una realidad, tal como lo indican las investigaciones realizadas en el país. El Instituto Nacional de Carnes (INAC) en conjunto con el Instituto Nacional de Investigación Agropecuaria (INIA) y la Universidad Estatal de Colorado (CSU), llevaron a cabo dos auditorías de Calidad de la Cadena Cárnica Vacuna (2003; 2008), que mostraron un descenso en la prevalencia de lesiones, siendo de $60.4 \%$ y $31.8 \%$ respectivamente. A pesar de que estos datos revelan un avance en el nivel de bienestar de los animales faenados, aún existen problemas.

Otro estudio realizado sobre 13 plantas de faena habilitadas del país, evidenció una prevalencia estimada de lesiones traumáticas en la media res de $48 \%$, lo que lleva a que por cada animal que se faenaba se estaba decomisando más de 1 $\mathrm{kg}$ de carne, equivalente a pérdidas de más de 3 millones de dólares americanos (U\$S 3.060.000), correspondientes al período de evaluación (Huertas, 2006). 
El país ha demostrado preocupación para evitar el sufrimiento innecesario, incorporando los principios del bienestar animal a lo largo de los distintos eslabones de la cadena cárnica. Se crea la Ley 18.471 sobre tenencia responsable de animales, decretos (Reglamento Oficial de Inspección Veterinaria, MGAP) sobre manejo, transporte y faena humanitaria de animales y se han realizado proyectos, investigaciones, difusión y capacitaciones por parte de distintas entidades a nivel nacional.

Dada la importancia de la prevalencia de hematomas como un indicador indirecto del bienestar de los animales destinados a producir carne para consumo humano, el objetivo de este trabajo fue evaluar y caracterizar los hematomas presentes en carcasas vacunas en algunas plantas de faena de Uruguay, habilitadas para la exportación. De esta forma se obtendrá, por un lado información sobre la evolución del manejo de animales previo a su faena, y por otra parte, se intentará relacionar las características de estas lesiones con la causa que las genera.

\section{MATERIALES Y MÉTODOS}

Selección de plantas y conformación de la muestra

La elección de las plantas se realizó por conveniencia

y los criterios de inclusión fueron por estar habilitadas para la exportación por el Ministerio de Ganadería Agricultura y Pesca (MGAP) y la Unión Europea y ser de las de mayor volumen de producción del país (según año agrícola).

Para la determinación del número de carcasas a observar por planta de faena y por visita, se decidió tomar un $25 \%$ del total de la faena del día de la visita.

Se realizaron visitas a 7 plantas de faena ubicadas en la zona sur del país, en los departamentos de Montevideo, Canelones y Cerro Largo. Durante los meses de septiembre y diciembre del año 2012, se evaluaron un total de 1.030 carcasas bovinas, incluyendo novillos, vaquillonas, toros y vacas.

Capacitación / entrenamiento de los observadores Previo al inicio de la investigación se llevó a cabo una instancia de entrenamiento de ambos integrantes del equipo para uniformar los criterios de observación a cargo de los profesionales veterinarios idóneos en el tema, pertenecientes al Instituto Nacional de Carnes (INAC).

\section{Clasificación de los hematomas}

La identificación, cuantificación y clasificación de los hematomas se realizó teniendo en cuenta la localización, área y profundidad de los tejidos afectados. Siguiendo los lineamientos de las auditorías de calidad de carne vacuna realizadas por INAC-INIA.

La carcasa se dividió en 8 diferentes zonas: el muslo, ubicado en la zona posterior de la res; región del flanco y costillar que representan la zona lateral; regiones de la grupa, lomo, dorso y cruz, que se localizan en el área dorsal de la carcasa; región de la paleta, que representa la zona delantera de la res (ver esquema).

a) Clasificación de hematomas según profundidad de los tejidos afectados:

Hematomas grado 1 - Superficial. Interesa únicamente tejidos subcutáneos. La lesión no afecta al tejido muscular subyacente. Puede presentar un sangrado mínimo.

Hematomas grado 2 - Medio. Involucra planos musculares intermedios, con evidencia de hemorragia importante.

Hematomas grado 3 - Profundo. Gran pérdida de tejido muscular. Interesa a su vez al tejido óseo, el cual puede presentar lesiones como fracturas.

b)- según área que abarca la lesión:

A1) hasta $18 \mathrm{~cm}$ de diámetro (aprox. $254 \mathrm{~cm} 2$ )

A2) hasta $30 \mathrm{~cm}$ de diámetro (aprox. $700 \mathrm{~cm} 2$ )

A3) más de $30 \mathrm{~cm}$ de diámetro. Puede incluir la totalidad de la región.

A4) generalizada. Cuando se evidencian 5 o más regiones de la carcasa afectadas por hematomas con una extensión equivalente a un A2 o A3. 
c)- según forma:

En base a la literatura, las distintas formas evidenciadas en este estudio se categorizaron en 4 grupos: forma "lineal", en "tranvía", formas "circulares / irregulares" incluidas en una única categoría. Finalmente, una última categoría representada por hematomas en forma de "coma", los cuales están representados por una figura que describe una línea curva, con un extremo ancho y el otro más fino, de un largo aproximado entre 20 y $30 \mathrm{~cm}$. Esta lesión característica no cuenta con antecedentes en la literatura, sin embargo, se incluye en el presente estudio, debido a su frecuencia de aparición.

\section{Análisis estadístico}

Los datos obtenidos durante la caracterización de las lesiones fueron incorporados a planillas electrónicas (Excel) para el análisis descriptivo, que se realizó con el paquete estadístico Stata Intercooler 11.2 (2009), de manera de obtener las frecuencias de cada variable considerada.

Las variables sexo y edad, se estudiaron relacionándolas con la manifestación de hematomas en la canal. Esta evaluación se llevó a cabo mediante la prueba de Chi Cuadrado.

\section{RESULTADOS}

Los resultados evidenciaron que de un total de 1030 reses observadas, el $44.4 \%$ (457 carcasas) presentaron algún tipo de hematoma. Este porcentaje se mantiene, en forma aproximada, en la mayoría de las plantas de faena, cuando se analizan individualmente (Figura 1). Exceptuando los establecimientos $\mathrm{F}$ y $\mathrm{G}$ con porcentajes de 26.9 $\%$ y $63.3 \%$ respectivamente.

Se registraron un total de 917 hematomas, lo que en promedio resulta en 0.89 hematomas por carcasa. Considerando únicamente las reses con lesiones, 228 presentaron un sólo hematoma (49.9 \%), mientras que 229 tuvieron 2 o más $(50.1 \%)$.

Localización de los hematomas en las carcasas

A punto de partida del total de hematomas registrados en las carcasas $(n=917)$, se realizó un gráfico representativo referente a la distribución de los mismos, teniendo en cuenta, la región de la canal en que se localizan y el grado de profundidad de la lesión (Fig 2). Las zonas más afectadas fueron la del muslo, costillar, flanco y región dorso-lumbar, con $31 \%, 19.7 \%, 19.6 \%$ y $15.3 \%$ respectivamente.

\section{Clasificación de los hematomas según área y grado de profundidad}

Respecto de la superficie que abarcan, sólo 5 carcasas (1.09\%) presentaron hematomas generalizados.

En cuanto a la profundidad, un $16.1 \%$ de las reses presentaron lesiones grado 2 y el $83.9 \%$ lesiones superficiales o grado 1 . No se detectaron hematomas severos, grado 3 , en ninguna de las carcasas evaluadas (Figura 3).

Los hematomas grado $2(n=126)$ son los que naturalmente afectan en mayor medida, debido a que implica lesiones en tejidos musculares. $\mathrm{Su}$ distribución en la res determina que la región más comprometida es la del muslo con $34.9 \%$, seguido por la zona dorso-lumbar y el flanco, con $22.2 \%$ y $21.4 \%$ respectivamente (Figura 4 ).

\section{Forma de los hematomas}

La mayor proporción encontrada fue de hematomas circulares e irregulares con un $93.1 \%$ sobre el total de lesiones $(n=917)$. Por otra parte, las distintas formas registradas presentaron una mayor frecuencia de aparición en zonas características de la canal (Figura 5). Los hematomas lineales se registraron mayormente en zonas de costillar, dorso-lumbar, flanco y paleta, con 56,4\%, 20\% y $10,9 \%$ respectivamente. En cuanto a los hematomas en forma de "coma" se localizó la totalidad en la región del costillar.

\section{Influencia de la edad y el sexo en la aparición de lesiones traumáticas.}

Utilizando la prueba de Chi Cuadrado al evaluar los animales faenados según edad y sexo, se encontraron diferencias significativas en la aparición de lesiones traumáticas $(\mathrm{p}<0,01)$, tal como se muestra en la Figura 6. 


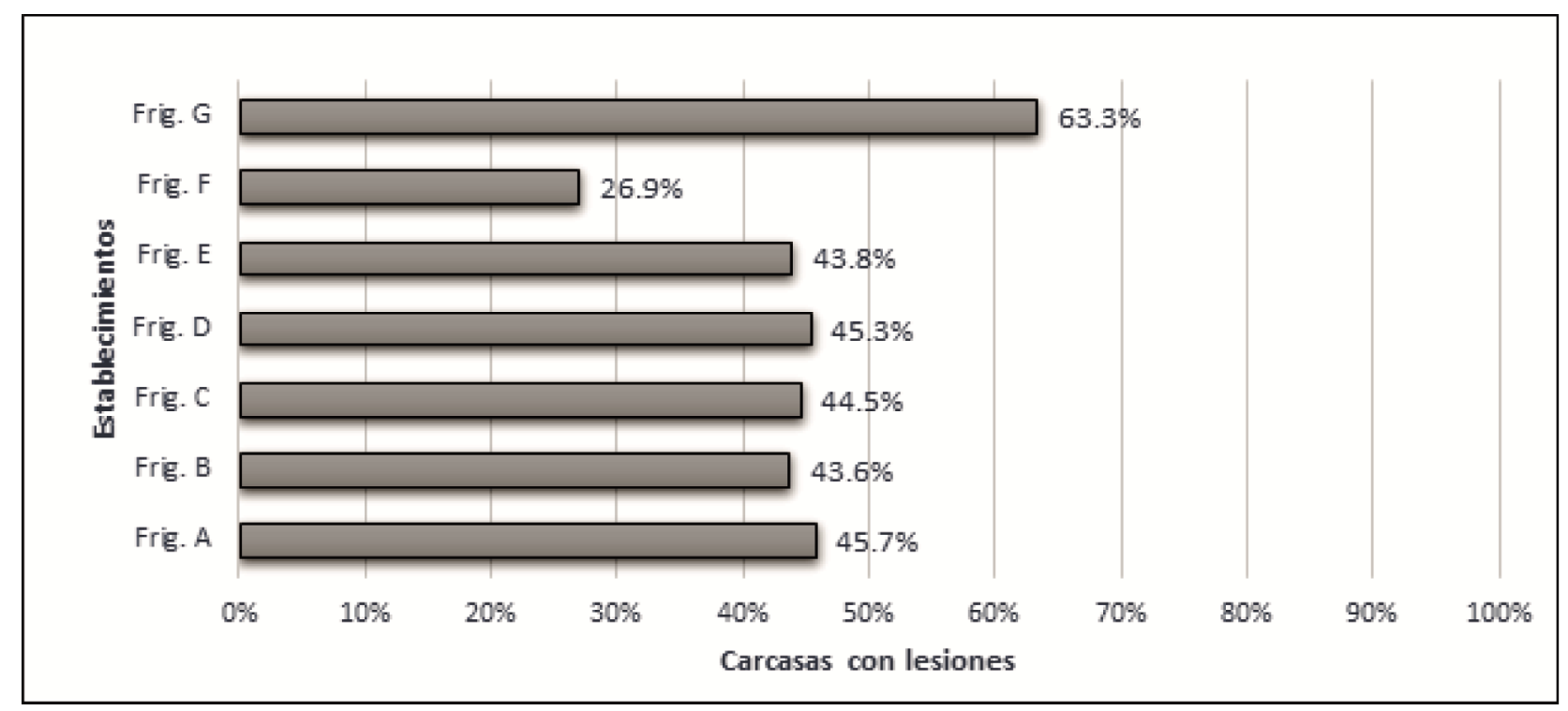

Figura 1. Porcentaje de carcasas que presentaron hematomas en cada establecimiento frigorífico. A los efectos de mantener la confidencialidad, las plantas se nombraron con letras.

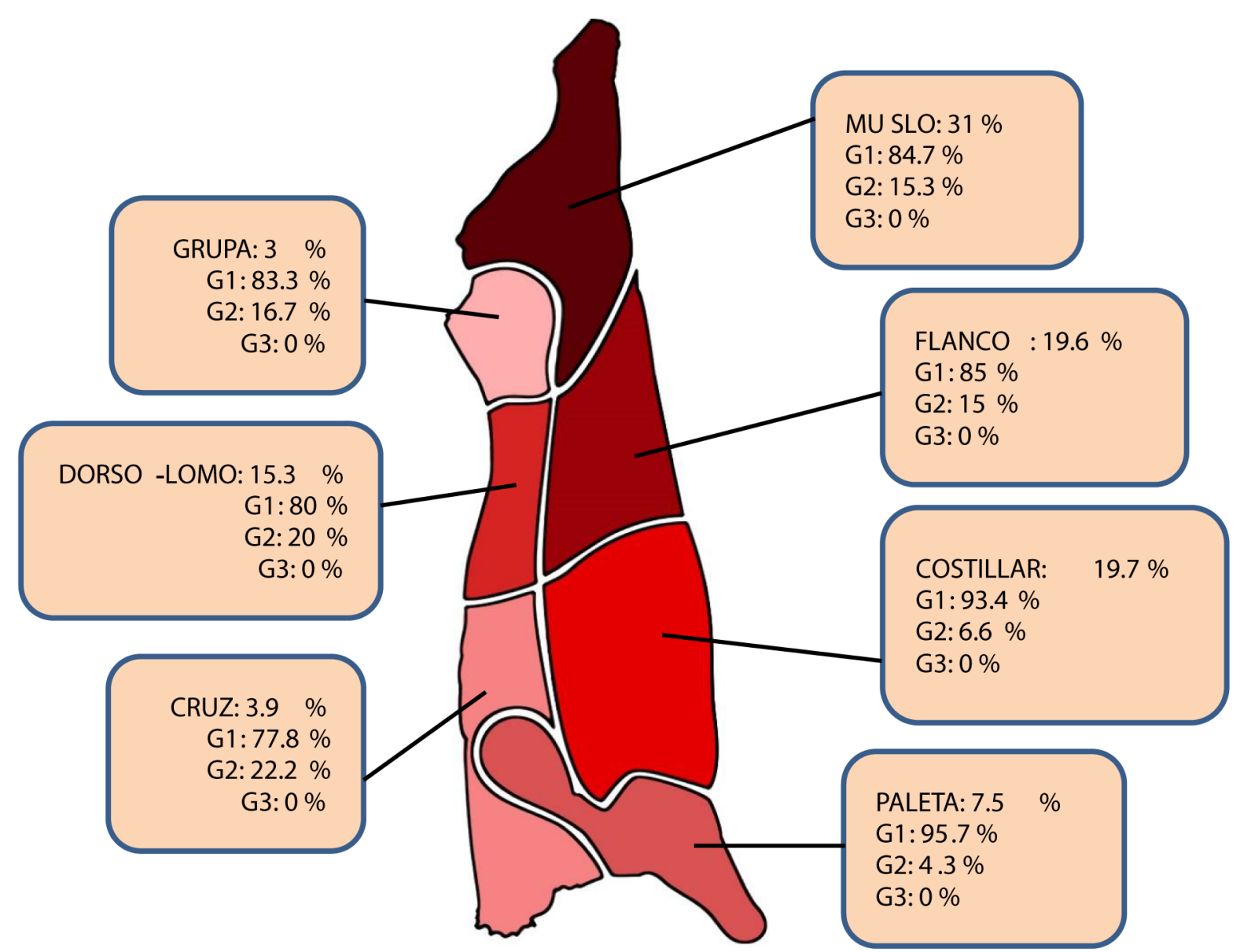

Figura 2: Distribución de hematomas según la región que involucra en la carcasa y profundidad de la lesión. 


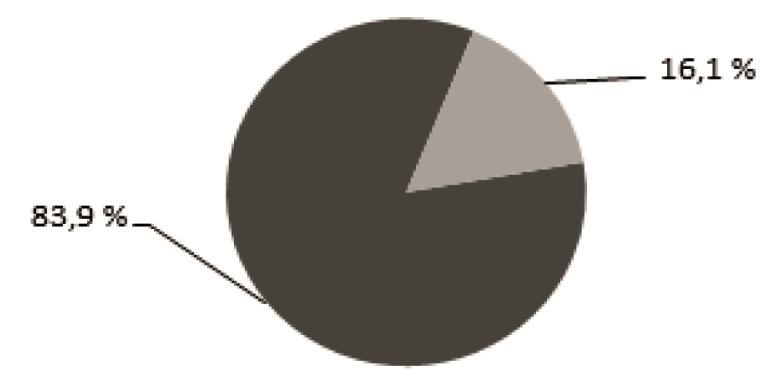

- Grado 1Figura 3. Porcentaje de hematomas 口 Grado 2 según grado de profundidad.

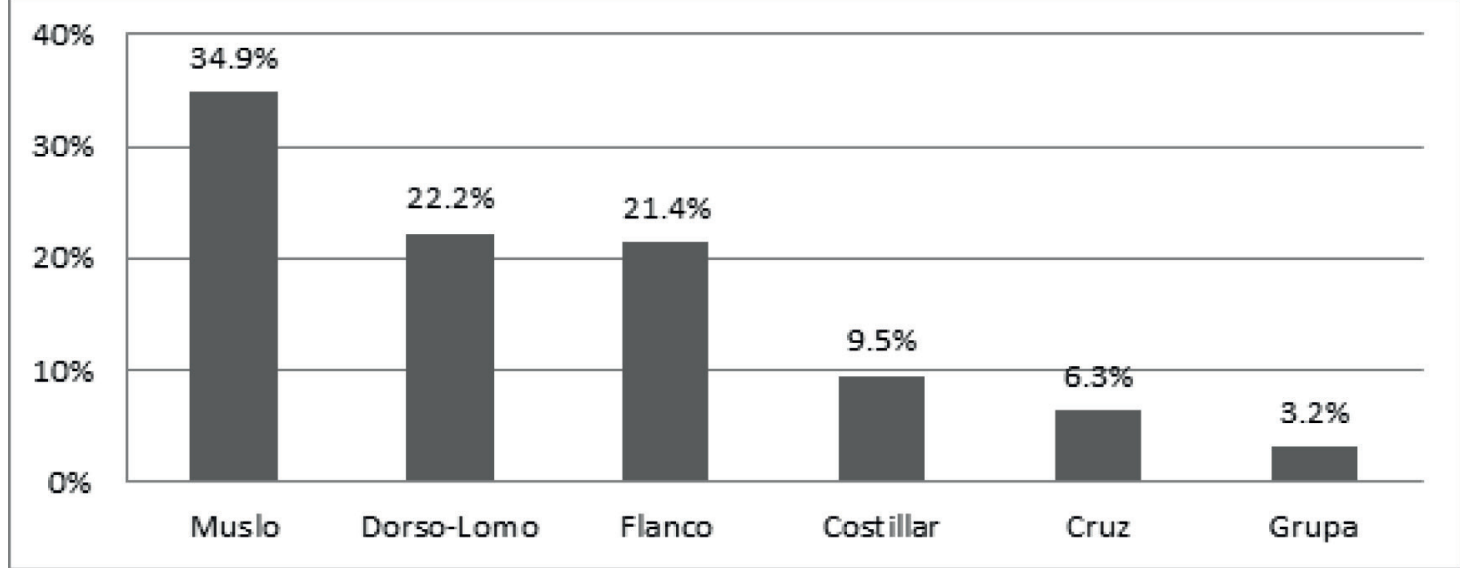

Figura 4. Distribución de hematomas grado 2 por región en la res.

\section{Circular / Irregular}

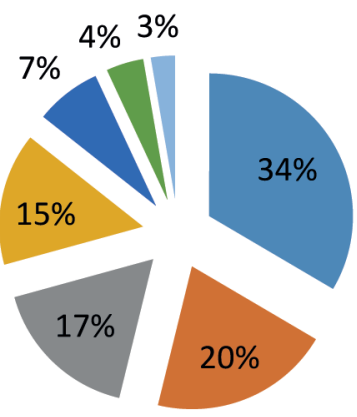

\section{Tranvía}

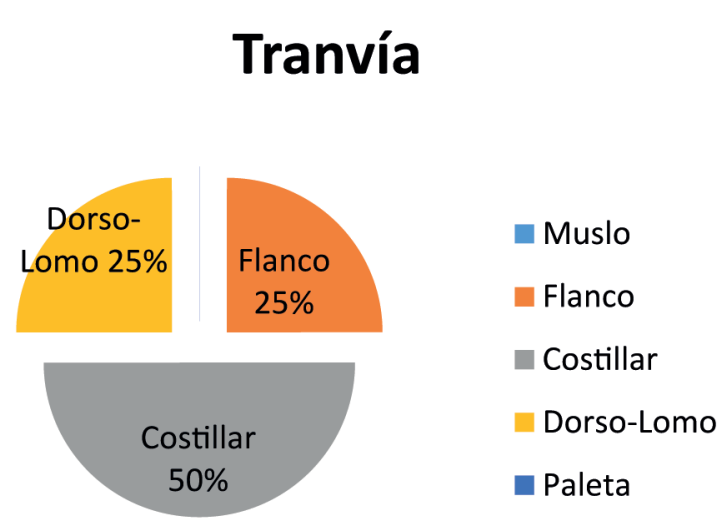

Muslo

- Flanco

Costillar

Dorso-Lomo

- Paleta

a Cruz

Grupa

\section{Lineal}

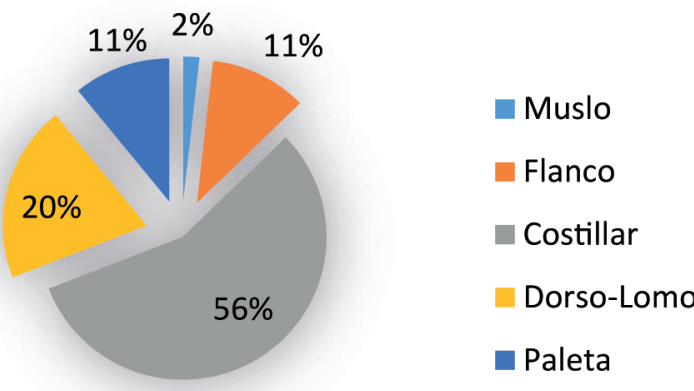

Coma

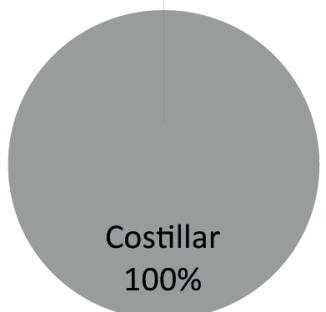

Muslo

Flanco

Costillar

Dorso-Lomo

- Paleta

Figura 5. Distribución en la carcasa de los distintos tipos de hematomas registrados. 

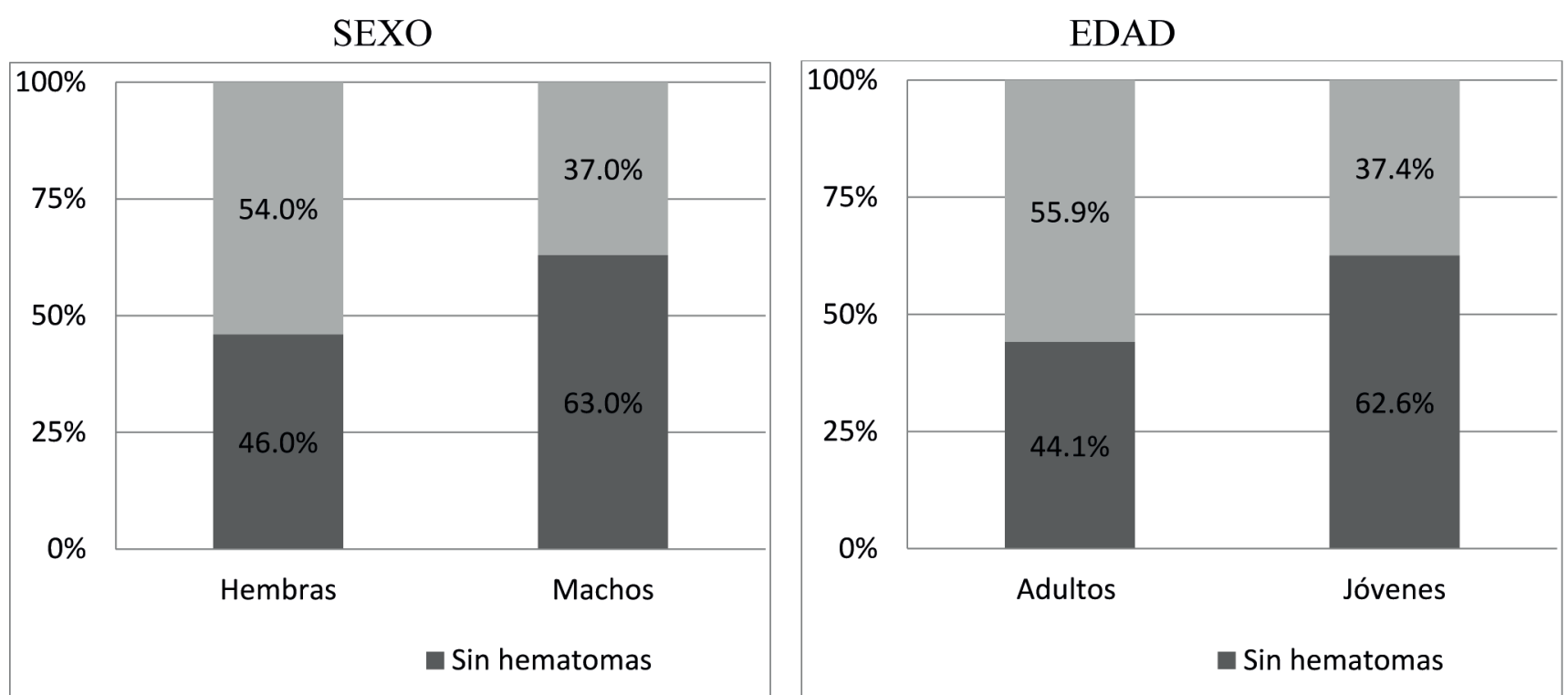

Figura 6. Porcentaje de hematomas según sexo y edad. Estos resultados refieren a los establecimientos A, C, D, E y F en los que se obtuvo información sobre la edad y sexo de los individuos faenados (773 carcasas en total).

\section{DISCUSIÓN}

La presentación de este tipo de lesiones traumáticas en las carcasas de bovinos en Uruguay, tiende a disminuir según lo demuestran los trabajos nacionales realizados por INACINIA-CSU (2003), Huertas (2006) y nuevamente INAC-INIA en su segunda auditoría (2008) con $60.4 \%, 48 \%$ y $31.8 \%$ respectivamente. Si bien los resultados del actual trabajo no acompañan dicho descenso, debemos considerar que los establecimientos evaluados no coinciden en su totalidad con las investigaciones anteriores.

Se observa que la mayoría de los establecimientos de faena presentaron una prevalencia similar a la media poblacional, sólo dos se alejaron de la misma. El $26.9 \%$ registrado en el establecimiento "F", pudo deberse a que el $100 \%$ de los animales faenados el día del estudio fueron novillos, que por sus características de edad y sexo, es menos probable encontrar este tipo de lesiones (Weeks et al., 2002; Strappini et al., 2010). En cuanto al alto porcentaje de hematomas evidenciado en el establecimiento "G", puede estar fundamentado por la alta cantidad de vacas viejas faenadas, las cuales son más susceptibles a la generación de hematomas (Yeh et al., 1978).
El número de lesiones por carcasa es un dato de gran valor al momento de evaluar el nivel de bienestar animal previo a la faena. El $50.1 \%$ de las carcasas con más de 2 hematomas, detectadas en el actual trabajo, son similares al $54 \%$ encontrado por Huertas (2006). Si consideramos a los hematomas como indicativo de dolor $\mathrm{y}$ sufrimiento experimentado por el animal en vida (Strappini et al., 2009), es de esperar que cuanto más cantidad presenten sus carcasas, peores fueron las condiciones a las que fueron sometidos antes de su muerte.

Así como la cantidad, la profundidad que alcancen las lesiones es un reflejo del nivel de dolor que experimentaron los animales. El $16.1 \%$ de carcasas constatadas con hematomas profundos que afectan las capas musculares, difiere con datos anteriores. Huertas (2006) detecta que el $21.5 \%$ de las reses sufrieron hematomas grado 2 y $2.9 \%$ lesiones grado 3. Por su parte, INAC-INIA (2008) encuentra que un $44.3 \%$ de las carcasas lesionadas manifestaron hematomas profundos. Tal vez la diferencia más destacable en el presente estudio es la ausencia de hematomas grado 3 (severos), los que por su naturaleza implican el mayor grado de dolor para los animales y mayores pérdidas de tejido muscular. 
El mayor porcentaje de contusiones se encontró en la zona del muslo, más específicamente en los músculos alrededor de la cola, lo que coincide con estudios nacionales (Huertas, 2006). Posiblemente, debido a la forma circular que presentaron la mayoría de los hematomas en esta zona, sean a causa de golpes contra estructuras duras dentro de los vehículos de transporte. Tal como destaca Hoffman \& Lühl (2012) en su estudio, altas densidades de animales en los camiones, rutas en mal estado y maniobras incorrectas del conductor, provocan pérdidas de equilibrio por parte de los animales y golpes sobre esta región. Otras de las zonas más afectadas fueron el costillar y el flanco, a lo cual Gallo et al. (2005) atribuyen a golpes contra estructuras duras (instalaciones o vehículos mal mantenidos), uso de instrumentos de manejo como palos, picanas o ataques por parte de otros animales (Blackshaw et al., 1987; Warriss, 1990).

Los hematomas localizados en el área dorso-lumbar pueden ser generados por las puertas tipo guillotina de los camiones de transporte de ganado que no abren totalmente o porque en algunos casos, los propios operarios las dejan caer sobre los animales para que éstos avancen (Grandin, 1997; Blackshaw et al., 1987, Huertas, 2006). Estas regiones de mayor afectación (muslo, dorso y lomo), generan preocupación en la industria cárnica nacional ya que involucra a los cortes de mayor valor comercial y los que conforman los grupos de cortes para exportación a los países más exigentes (Huertas, 2006).

En los 5 animales con evidencia de traumatismos generalizados (1.09\%), las lesiones se localizaron principalmente en las regiones del costillar, flanco, cruz, dorso y lomo, posiblemente a causa de caídas durante el transporte. Si un daño severo ocurre y una larga porción de la carcasa está completamente lesionada, esto puede indicar que el animal fue pisoteado por los demás luego de haber sufrido una caída en el camión (Strappini et al., 2009).

Los resultados de la última auditoría de faena humanitaria realizada por INAC (2009), destacan que la mayor problemática en nuestro país es el maltrato de los animales por parte de los operarios.
Una estrategia para evaluar este factor, es el estudio del patrón que manifiestan los hematomas, debido a la estrecha asociación existente entre la forma de los mismos y el evento causal que los genera (Grandin, 2000). Aquellos clasificados como lineales o en tranvía, presentan una forma similar al objeto con el que se inflige la lesión, como es el caso de palos o varas (Strappini et al., 2011; McNally \& Warriss, 1996; Weeks et al., 2002). Si relacionamos su forma con su localización, la cual fue principalmente en regiones de costillar, dorsolumbar, flanco y paleta, podemos deducir que los animales del presente estudio han sido sometidos a un mal uso y abuso de instrumentos para hacerlos avanzar o retroceder.

En cuanto a los hematomas en forma de "coma", el patrón que presentan y su localización en la canal (región del costillar), permiten relacionarlos con el uso del conocido "rebenque". Éste es un instrumento procedente de países como Uruguay y Argentina, constituido por un trozo de cuero vacuno unido a un mango, diseñado para utilizarlo sobre los animales de manera de estimular su movimiento.

Las características de cada sexo hace que existan diferencias físicas como la cobertura de grasa, y el espesor de la piel, afectando la susceptibilidad a la generación de hematomas de mayor o menor severidad con impactos de similar fuerza (Weeks et al., 2002; Strappini et al., 2010). En general las hembras de las especies de animales de producción presentan más hematomas que los machos, lo que coincide con los resultados obtenidos en este trabajo. Así mismo, animales viejos y maduros son más propensos a manifestar hematomas frente a situaciones traumáticas en comparación con animales jóvenes (Yeh et al., 1978).

De acuerdo al número y características de los hematomas encontrados, podemos concluir que a pesar de haber tenido avances sustanciales en el país, el nivel de bienestar de los animales, respecto de este factor estudiado, aún debe estar sujeto a mejoras. La reducción en la aparición de hematomas grado 2, así como la ausencia de hematomas grado 3 , evidencian una mayor concientización en el personal que maneja los animales, demostrando 
más cuidado en el trato. Sin embargo, la presencia de carcasas con lesiones generalizadas, evidencian por el contrario posibles errores del personal durante el transporte. La forma de manejo del vehículo, las características y condiciones de las rutas utilizadas, la mezcla de animales de distintos orígenes y/o categorías, son algunos de los factores que pueden influir sobre el nivel de bienestar animal y la generación de hematomas, y que por lo tanto deberían ser objeto de estudios más específicos.

La relación entre la forma de los hematomas y su localización en la carcasa, fue una herramienta de gran valor que permitió detectar en forma indirecta que los operarios aún mantienen prácticas inadecuadas de manejo de animales. Si bien las campañas de capacitación y difusión han generado un impacto positivo, los resultados obtenidos en este trabajo muestran que aún se necesita continuar en esta línea, y en algunos casos hasta puede ser necesaria una re-capacitación al personal involucrado directa o indirectamente en la cadena cárnica.

Es fundamental que todas las personas involucradas en la cadena de producción cárnica (pública y privada), manejen el concepto de que los animales son ante todo seres vivos, capaces de sentir dolor y sufrimiento, y como tales les debemos respeto. Por otra parte, debemos lograr un entendimiento sobre el impacto y el valor que tienen las buenas prácticas de manejo y conceptos de bienestar animal, sobre el producto final obtenido, apuntando a la economía nacional y a nuestro prestigio a nivel mundial como exportadores de carne de calidad.

\section{REFERENCIAS}

Blackshaw, J. K, Blackshaw, A. W., Kusano, T. (1987). Cattle Behavior in a sale yard and its potential to cause bruising. Australian Journal of Experimental Agriculture; 27:753-757.

Castro L., Robaina R. (2003). Manejo del ganado previo a la faena y su relación con la calidad de la carne. Montevideo, Uruguay. Instituto Nacional de Carnes (INAC) $31 \mathrm{p}$.
Cervieri, V., Rovira, F., Castro, L. (2010). Bienestar Animal. Su rol en la producción de carne de calidad. INAC Serie técnica No 47. Montevideo, Uruguay. Instituto Nacional de Carnes. 135p.

Gallo, C. (2004).Alternativas para mejorar el manejo prefaena de bovinos: manejo para una producción de calidad". Durazno, Kiyu, Tacuarembó, Uruguay; p 8-16.

Gallo, C., Warris, P., Knowles, T., Negron, R., Valdés, A., Mencarini, I. (2005). Stocking densities used to transport cattle to slaughter in Chile. Archivos de Medicina Veterinaria; 37:155-159.

Grandin, T. (1997). The design and construction of facilities for handling cattle. Livestock Production Science; 49: 103-119.

Grandin, T. (2000). Livestock handling and transport. $2^{\mathrm{a}}$ ed. Wallingford; CABI. 449p.

Gracey, J.F., Collins, D.S., Huey, R.J. (1999). Meat Higyene. 10a Edición. W.B. Saunders Ltda. Londres. $768 p$.

Hoffman, D. E., Spire, M. F., Schwenke, J. R., \& Unruh, J. A. (1998). Effect of source of cattle and distance transported to a comercial slaughter facility on carcass bruises in mature beef cows. Journal of American Veterinary Medicine Association, 212, 668-672.

Hoffman, L., Lühl, J. (2012). Causes of cattle bruising during handling and transport in Namibia. Meat Science; 92: 115-124.

Huertas, S. M. (2004). Puntos críticos que afectan el bienestar de los animales. Recomendaciones para mejorar la calidad de la carne. Conferencia "Bienestar animal: alternativas de manejo para una producción de calidad". Durazno, Kiyu, Tacuarembó, Uruguay; p 17-22. 
Instituto Nacional de Carnes; Instituto Nacional de Investigaciones Agropecuarias; Colorado State University. (2003). Auditoría de Calidad de Carne Vacuna. Uruguay; INAC-INIA-CSU. 23p.

Instituto Nacional de Carnes; Instituto Nacional de Investigaciones Agropecuarias; Colorado State University. (2008). 2a Auditoría de Calidad de Carne Vacuna.Uruguay; INAC-INIA-CSU. 46p.

Marshall, B. (1977). Bruising in cattle presented for slaughter. New Zealand Veterinary Journal; 25: 8386.

McNally, P., Warriss, P. (1996). Recent bruising in cattle at abattoirs. TheVeterinary Record; 138: 126128.

Ministerio de Ganadería Agricultura y Pesca. (1983). Decreto 369/983. Reglamento Oficial de Inspección Veterinaria de Productos de Origen Animal. Disponible en: www.mgap.gub.uy. Fecha de consulta: 19 de Septiembre de 2012.

Ministerio de Ganadería Agricultura y Pesca, DGSG (2012). Lanzó la Resolución DGSG/No152/012 sobre "Bienestar Animal para Faena - exigencias UE”. Disponible en: http://www.mgap.gub.uy. Fecha de consulta: 28 de noviembre de 2012 .

Strappini, A., Metz, J., Gallo, C., Kemp, B. (2009). Origen y valoración de machucones de bovinos de carne en faena. The Animal Consortium; 3(5): 728736.

Strappini, A., Frankena, K., Metz, J., Gallo, C., Kemp, B. (2010). Prevalence and risk factors for bruises in Chilean bovine carcasses. Meat Science; 86: 859-864.

Strappini, A., Frankena, K., Metz, J., Gallo, C., Kemp, B. (2011). Characteristics of bruises in carcasses of cows sourced from farms or from livestock markets. The Animal Consortium; 1-8.

Warriss, P. (1990). The handling of cattle preslaughter and its effects on carcass and meat quality. Applied Animal Behaviour Science; 28: 171-186.
Weeks, C., McNally, P., Warriss, P. (2002). Influence of the design of facilities at Auction markets and animal handling procedures on bruising in cattle. The Veterinary Record; 150: 743-748.

Yeh, E., Anderson, B., Jones, P., Shaw, F. (1978). Bruising in cattle transported over long distances. The Veterinary Record; 103: 117-119.

FAO (2001) www.fao.org Fecha de consulta: 14 de octubre de 2014.

INAC (2003) www.inac.gub.uy Fecha de consulta: 16 de septiembre de 2014. 